\title{
Structure and Electrochemical Behavior of Aromatic Thiol Self-Assembled Monolayers on Au(111)
}

\author{
Jaegeun Noh," Hajung Park, Youngdo Jeong, and Seungwook Kwon \\ Department of Chemistry, Hanyang University, Seonl 133-791, Korea. "E-mail:jgnoh@hamang.ackr \\ Received October 10, 2005
}

\begin{abstract}
The surface structure and electrochemical behavior of self-assembled monolayers ( $\mathrm{S} \Lambda \mathrm{Ms}$ ) formed by aromatic thiols on $\Lambda u(111)$ were investigated by scanning tunneling microscopy (STM) and cyclic voltammetry. Benzenethiol (BT) forms disordered phases on $\Lambda u(111)$ which are composed of many bright domains, while benzyl mercaptan (BM), with a methylene unit between the aromatic group and sulfur atom, forms twodimensional ordered $\mathrm{S} \Lambda \mathrm{M}$ s on $\Lambda \mathrm{u}(111)$. In addition, two phase-separated domains consisting of disordered and ordered phases were observed in binary $S \Lambda$ Ms formed from a $1: 1$ mixed ethanol solution of BT and BM. From STM and CV measurements, we found that the blocking efficiency of aromatic thiol $\mathrm{S} \Lambda \mathrm{M}$ s coated on an $\Lambda u(111)$ electrode for an electron transfer reaction decreases as the structural order of the $\$ \Lambda \mathrm{Ms}$ increases. Molecular-scale STM and CV results obtained here will be very useful in designing functional $\mathrm{S} \Lambda \mathrm{M}$ s for further applications, such as the improvement of corrosion passivation of $\Lambda \mathrm{u}(111)$ on an aromatic thiolmodified $\Lambda u(111)$ surface.
\end{abstract}

Key Words : Benzenethiol, Benzyl mercaptan, Self-assembled monolayers, Scanning tunneling microscopy, Cyclic voltametry

\section{Introduction}

Self-assembled monolayers (SAMs) formed by the spontaneous adsorption of organothiols and disulfides on metal surfaces have drawn considerable attention in both basic research and various technological applications. ${ }^{1-8}$ Alkanethiol SAMs have been examined most extensively using various surface-sensitive techniques. As a result, fundamental aspects, such as the self-assembly mechanism, molecular packing structure, and thermal and long-term stability of alkanethiol SAMs, have been thoroughly revealed. ${ }^{12.8}$ In recent years, aromatic thiol SAMs have come to receive attention because of their high conductivity and strong structural rigidity compared to conventional alkanethiol SAMs, making them interesting for molecular electronics and electrode modification. ${ }^{9-11}$ It has been realized that the electronic properties of SAMs strongly depend on molecular orientations, adsorption conditions, and intermolecular interactions. Therefore, the control of the structural ordering of aromatic thiol SAMs has become a crucial issue in tailoring various SAM properties. It has been demonstrated that the degree of order for aromatic thiol SAMs can be remarkably enhanced by increasing the number of benzene rings in the molecular backbone. ${ }^{12}$ Structural order can be increased by introducing a methylene unit between the benzene ring and sulfur headgroup. ${ }^{13}$

To our knowledge, there are no reports describing the relationship between the structural order or disorder of aromatic thiol SAMs and their electrochemical behavior. In addition, understanding the phase behaviors of binary SAMs formed by two similar aromatic thiols is essential in tailoring nanoscale surface properties. The detailed information should help us determine how to precisely control the interface properties on the basis of the controlled SAM structures to make them more suitable for practical applications. In order to achieve our study goal, we have characterized the structures and electrochemical behaviors of benzenethiol (BT) SAMs, benzyl mercaptan (BM) SAMs, and binary SAMs of $\mathrm{BT}$ and $\mathrm{BM}(1: 1$ ratio $)$ using scanning tunneling microscopy (STM) and cyclic voltammetry (CV).

\section{Experimental Section}

Benzenethiol (BT) and benzyl mercaptan (BM) were purchased from Tokyo Kasei Kogyo Co., Ltd. and were used without further purification. Au(111) substrates were prepared by thermal evaporation onto mica as described previously. ${ }^{14}$ SAM samples were prepared by dipping the gold substrates in a $1 \mathrm{mM}$ ethanol solution of each aromatic thiol for $2 \mathrm{~h}$. Binary SAMs of BT and BM were prepared by immersing the gold substrates in a $1 \mathrm{mM}$ ethanol solution of BT and BM (1: 1 mixture) for $2 \mathrm{~h}$. After the SAM samples were removed from the solutions, the samples were thoroughly rinsed with a pure ethanol solution to remove weakly adsorbed molecules from the SAM surface.

STM measurements were performed using a NanoScope E (Veeco, Santa Barbara, CA) with a commercial Pt/Ir (80/20) tip. All STM images were acquired using the constant current mode in air at room temperature. The tunneling current $\left(\mathrm{I}_{1}\right)$ and bias voltage $\left(\mathrm{V}_{\mathrm{b}}\right)$ were $0.27 \mathrm{nA}$ and $500 \mathrm{mV}$, respectively; the imaging conditions for all STM images were the same. Electrochemical experiments were carried out with the BAS-100, employing a three-electrode cell. The gold electrode coated with aromatic thiols was used as the working electrode, and a platinum wire and $\mathrm{Ag} / \mathrm{AgCl}(\mathrm{KCl}$ sat.) were used as the counter and the reference electrodes, 
respectively. All solutions consisted of $1 \mathrm{mM} \mathrm{K} 4\left[\mathrm{Fe}(\mathrm{CN})_{6}\right]$ and $1 \mathrm{M} \mathrm{KNO}_{3}$ aqueous electrolyte and were thoroughly deoxygenated by purging with nitrogen gas for $20 \mathrm{~min}$ prior to each experiment.

\section{Results and Discussion}

Controlled two-dimensional structures of aromatic thiol SAMs on $\mathrm{Au}(111)$ can be obtained using BT and BM molecules. Previous spectroscopy studies of BT SAMs revealed that the benzene ring of BT on gold is oriented parallel to the surface, ${ }^{15}$ and STM imaging showed that BT SAMs usually have disordered phases consisting of many bright domains. ${ }^{13}$ Figure 1a is a typical STM image of BT SAMs which shows disordered phases containing these bright domains. This type of molecular feature was also observed in previous STM results. ${ }^{12}$ It is thought that the formation of these bright domains is one of intrinsic properties of BT SAMs because similar surface structures were always obtained from BT SAM samples regardless of the various experimental conditions used for SAM preparation. The bright domains may be due to a change in molecular orientation derived from a higher molecular packing density of adsorbed BT molecules.

On the other hand, previous $\mathrm{STM}^{13}$ and theoretical calculation ${ }^{16}$ studies revealed that a $\mathrm{BM}$ molecule with a methylene unit between the benzene ring and sulfur atom can form a two-dimensional ordered phase with a $(\sqrt{3} \times$ $\sqrt{3}) \mathrm{R} 30^{\circ}$ structure, suggesting that the methylene unit plays an important role in enhancing the molecular ordering of aromatic thiol SAMs on Au(111). However, a recent STM study reported that BM molecules would not form ordered SAMs, even though they used similar experimental conditions for SAM preparation. " Moreover, electron energy loss spectroscopy (EELS) results suggested that BM molecules form ordered SAMs that can be described as $(\sqrt{7} \times$ $\sqrt{7}$ )R $19.1^{\circ}$ structures at saturation coverage. There remains a discrepancy between the two results for the lattice structure of BM SAMs obtained from STM and EELS measurements. In contrast to the recent STM and EELS results, however, our high-resolution STM image in Figure Ib for the BM

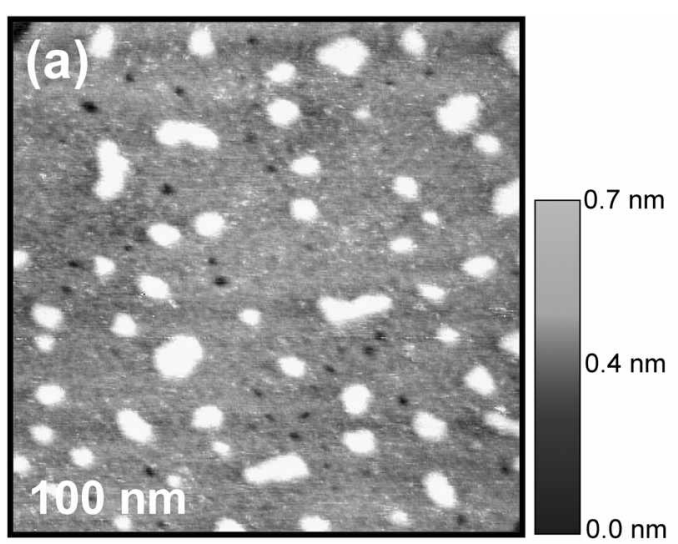

SAMs shows the formation of well-ordered phases with a $(\sqrt{3} \times \sqrt{3}) \mathrm{R} 30^{\circ}$ lattice structure, which strongly supports the STM results obtained by Tao et $a l^{13}$ Interestingly, it was also found that while SAMs of arenethiol (with three benzene rings in the molecular backbone) have clear domain orientation and boundaries, ${ }^{17}$ BM SAMs (with one benzene ring) have no clear domain orientation or boundaries. From this result, it is reasonable to assume that the adsorption geometry of the $\mathrm{BM}$ molecule is more perpendicular than that of the arenthiol molecule, because molecules with a tilted adsorption geometry from the surface normal usually show clear domain orientation and boundaries, as in the case of alkanethiols (with a tilt angle of about $30^{\circ}$ from the surface normal). ${ }^{7.18}$ Therefore, it is clear that the formation of domain orientation and boundaries are strongly related to the three-fold gold symmetry and tilt structure of adsorption molecules.

Binary SAMs composed of two different thiols are useful for controlling the properties of metal surfaces by properly choosing the type and ratio of the two thiol species. Binary SAMs with a nanometer-scale structure can be achieved by coadsorption from a solution containing two alkanethiols, ${ }^{19}$ adsorption of unsymmetric disulfide, ${ }^{20}$ and an exchange process. ${ }^{21}$ It has been revealed that phase-separated binary alkanethiol SAMs can only be formed when the chain length difference between the two alkanethiols is larger than four carbons. ${ }^{22}$ However, there are currently few reports on phase behaviors of mixed aromatic thiols on gold surfaces. As one approach to understanding the phase behaviors, BT and BM molecules, as simple molecular systems of aromatic thiols, were used for the preparation of binary SAMs on Au(111). Interestingly, in contrast to the phase behaviors of alkanethiols on gold, we observed two phase-separated domains with disordered and ordered structures ( $A$ and $B$ phases in Figure $2 \mathrm{a}$ and $2 \mathrm{~b}$ ) despite the small differences in the chemical structures of BT and BM. On the basis of the molecular behaviors of BT and BM for SAM formation (Figures 1a and 1b), it is reasonable to consider that ordered domains were formed by the adsorption of $\mathrm{BM}$ molecules and the disordered domains were mainly originated from the adsorption of BT molecules. However, the disordered phases

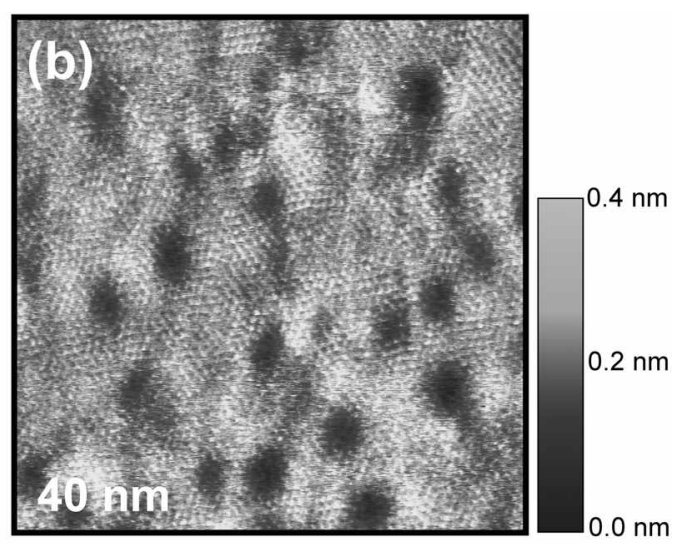

Figure 1. STM images of (a) BT and (b) BM SAMs on $\Lambda u(I, 1)$ formed after intmersion for $2 \mathrm{~h}$ in I mM ethanol solution. 

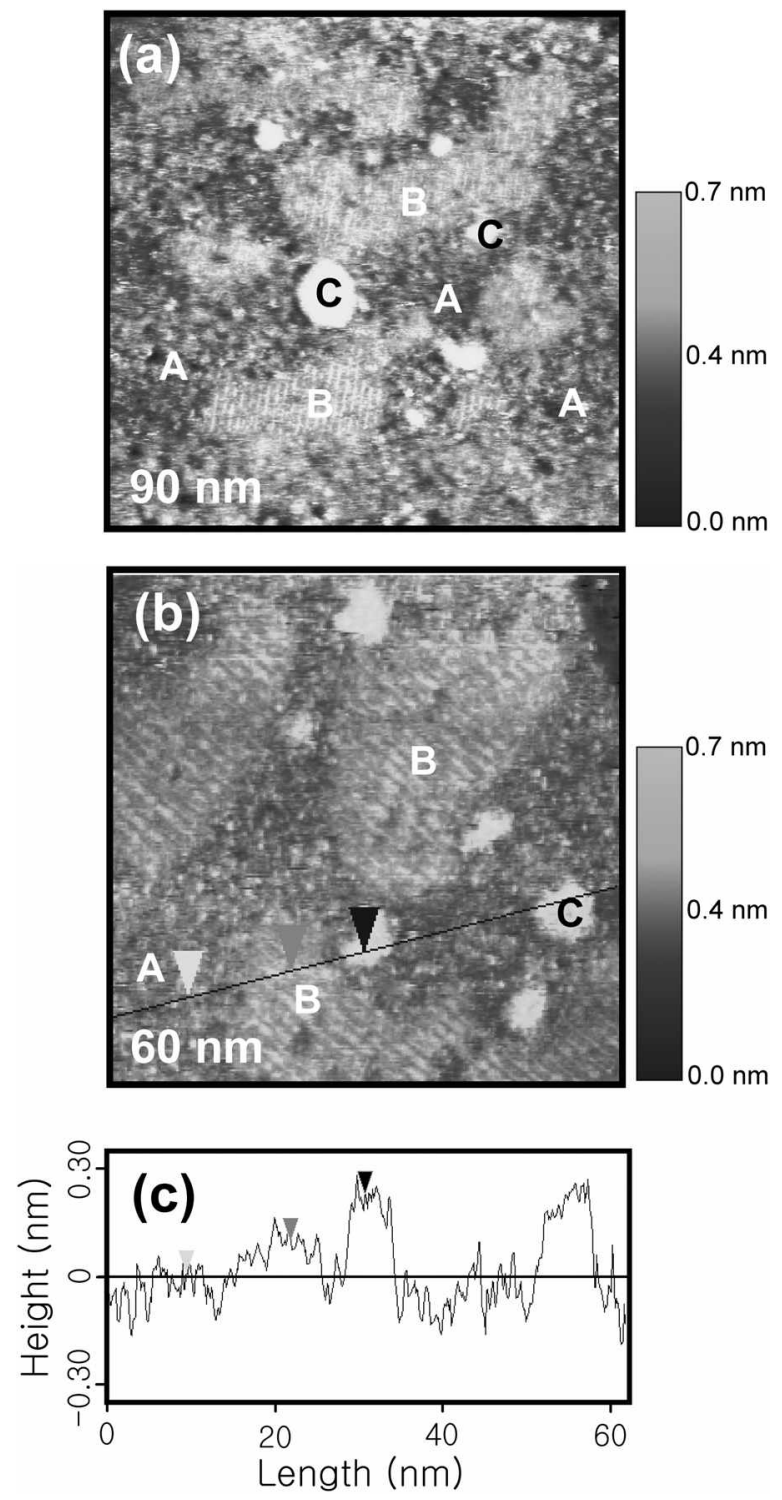

Figure 2. (a), (b) STM images showing phase separation of binary SAMs of BT and BM (1: 1 mixing) on Aul(111). (c) Height profile was obtained from along with the black line on the STM image of (b).

with many small aggregations (A phases in Figure $2 \mathrm{a}$ and $\mathrm{b}$ ) formed by coadsorption of BT and BM molecules were slightly different from those observed from singlecomponent BT SAMs (see the dark areas in Figure la, except bright domains). Therefore, even though the disordered phases formed from the mixed solution of BT and BM mainly result from the adsorption of $\mathrm{BT}$, it is likely that a small portion of $\mathrm{BM}$ molecules can also coexist in these phases.

Similar bright domains ( $C$ phases) shown in Figure 2a and $2 \mathrm{~b}$, as observed in BT SAMs (Figure 1a), were also found in the binary SAMs. Hence, we assume that such bright domains originated from BT molecules. Height profile of Figure $2 \mathrm{c}$ was obtained from along with the black line on the STM image of Figure $2 b$. This height profile clearly shows that three domains ( $\mathrm{A}, \mathrm{B}$, and $\mathrm{C}$ phases) have different turneling currents and the height differences between each domain were measured to be approximately $1.3 \AA$. It is noteworthy to mention that such height differences are mainly due to a variation in molecular orientations of adsorbed molecules as well as molecular components. The fraction of the areas of $\mathrm{A}, \mathrm{B}$, and $\mathrm{C}$ domains to the total surface area were measured to be approximately $52 \%, 45 \%$, and $3 \%$, respectively. However, we found that the formation of domains and the fraction of domain area in SAMs prepared from the binary mixture of BT and BM remarkably depend on molecular ratio of two components and immersion time. For instance, when we used a $2: 8$ mixed solution of $\mathrm{BT}$ and $\mathrm{BM}(2 \mathrm{~h}$ immersion) or after longer immersion than $4 \mathrm{~h}$ in a $1: 1$ mixed solution, we could only obtain single-component BM SAMs (Data not shown here). From this result, it is suggested that the formation of twodimensional ordered SAMs due to the adsorption of BM molecules is much more thermodynamically favorable than the formation of disordered phases resulting from BT molecules. Such phase behavior is mainly driven by the enhancement of van der Waals interaction between aromatic rings. Due to the above reasons, phase behaviors of the binary mixture are much more complicated than we expected. Systematic studies on these lines are now in progress and will be published elsewhere on separated paper.

From this study, we realized from a nanoscopic viewpoint that the structural characteristics of aromatic thiol SAMs can be controlled by the coadsorption of BT and BM molecules with the appropriate molecular ratio and immersion time. We would like to emphasize that the surface properties of organic monolayers on gold surfaces can be easily modifjed using the individual intrinsic molecular features of BT and $\mathrm{BM}$ molecules for SAM formation.

To understand the relationship between the surface structure and electrochemical behavior of aromatic thiol SAMs on gold, we examined electrochemical behaviors according to the structural changes of single-component $\mathrm{BT}$ and $\mathrm{BM}$ SAMs, and binary SAMs formed by BT and BM molecules. Figure 3 shows cyclic voltamograms of (a) bare-Au electrode, and of the Au electrodes modified with SAMs of (b) BT, (c) BT and BM (1: I mixing), and (d) BM in $1 \mathrm{mM}$ $\mathrm{K}_{4}\left[\mathrm{Fe}(\mathrm{CN})_{6}\right]$ and $1 \mathrm{M} \mathrm{KNO}_{3}$. The peak currents $\left(\mathrm{I}_{\mathrm{p}}\right)$ for $(\mathrm{a})$, (b), (c), and (d) were measured to be 40.1, 3.0, 7.1, and 8.6, respectively. Figure 3 a shows a well-defined CV characteristic of a diffusion limited redox process of the bare $\mathrm{Au}$ electrode. All aromatic SAMs coated on Au electrode efficiently blocked the electrode reaction of ferrocyanide. In particular, BT SAMs with the disordered structure show more effective blocking properties than the BM SAMs with the ordered structures. The blocking efficiency for the binary SAMs of BT and BM with the disordered and ordered structures lies roughly in between the single-component BT and BM SAMs. From this result, we found that the blocking efficiency of aromatic thiol SAMs decreases as the structural order of the SAMs increases. In the case of ultra-thin film (with a film thickness of less than $1 \mathrm{~nm}$ ), it is suggested that the electron transfer reaction between a solution species and 


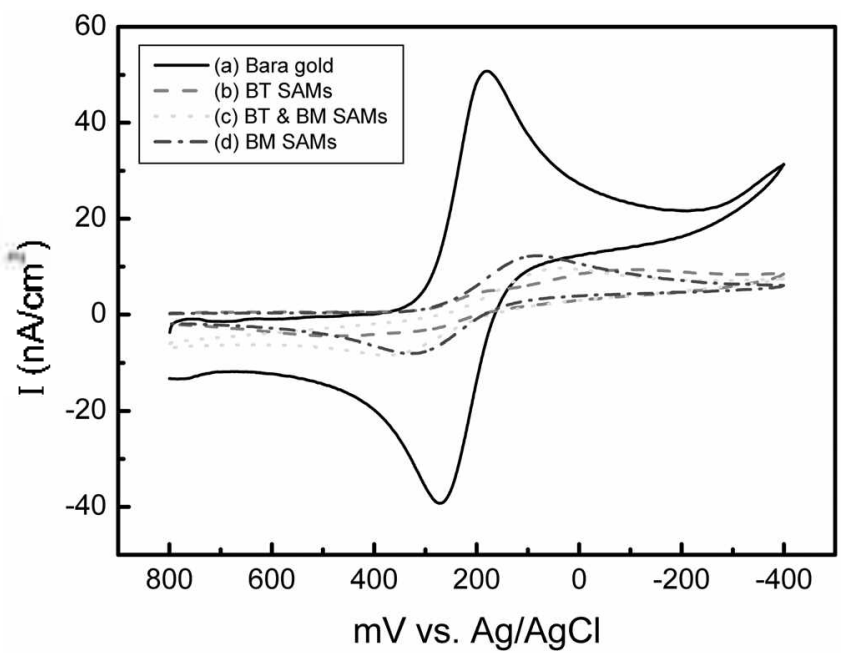

Figure 3. Cyclic voltamograms of (a) bare, (b) BT-, (c) BT and BM (1:1 mixing)-, and (d) BM- modified All electrodes in $1 \mathrm{mM}$ $\mathrm{K}_{4}\left[\mathrm{Fe}(\mathrm{CN})_{6}\right]$ containing $1 \mathrm{M} \mathrm{KNO}{ }_{3}$.

the electrode occurs much easier in the ordered monolayers than in the disordered monolayers. Our molecular-scale STM results will be very useful in the improvement of corrosion passivation of $\mathrm{Au}(11 \mathrm{I})$ in the aromatic thiolmodified Au(111) surface.

\section{Conclusion}

Molecular-scale STM imaging reveals that the binary SAMs on Au(111) formed by the adsorption of BT and BM molecules in ethanol solution have two phase-separated domains composed of disordered and ordered phases. Despite the small differences in the chemical structures of BT and BM, two phase-separated domains were forned, as opposed to what is seen with alkanethiols. From STM and $\mathrm{CV}$ measurements, it was found that the blocking efficiency of aromatic thiol SAMs coated on a Au(II1) electrode for the electron transfer reaction decreases as the structural order of the SAMs increases. Our results will be very useful in designing functional organic thin films for further technical applications, such as surface passivation, biointerfaces, and molecular electronics.

Acknowlergements. This work was supported by the research fund of Hanyang University (HY-2003).

\section{References}

1. Love, J. C.; Estroff, L. A.; Kriebel, J. K.; Nuzzo, R. G.; Whitesides, G. M. Chem. Rev, 2005, 105, 1103.

2. Schreiber, J. J. Phys.: Condens, Matter 2004, 16, R88I.

3. Noh, J.; Kato, H. S.; Kawai, M.; Hara, M. J. Ph:s. Chem. B 2002, $106,13268$.

4. Noh, J.; Konno, K.; Ito, E.; Hara, M. Jpn. J. Appl. Phts. 2005, 44. 1052.

5. Han, S. W. Bull. Korean Chem. Soc. 2005, 26, 463.

6. Chung C.; Lee, M. Bull. Korean Chem. Soc. 2004, 25, 1461.

7. Sung, M. M.; Yun, W. J.; Lee, S. S.; Kim, Y. Bull. Korean Chem. Soc: $2003,24,610$.

8. Noh, J.; Hara, M. Langmuir 2002, 18, 1953.

9. Tour, J. M. Ace. Chem. Res. 2000, 33, 791.

10. Zehner, R. W.; Parsons, B. F.; Hsung, R. P.; Sita, L. R. Lamgmir $1999,15,1121$.

11. Baunach, T.; Kolb, D. M. Anal, Bioanal. Chem, 2002, $373,743$.

12. Dhirani, A. A.; Zehner, R. W.; Hsung, R. P.; Guyot-Sionnest, P; Sita, L. R. J. Am. Chem. Soc. 1996, /18, 3319.

13. Tao, Y.-T.; Wu, C.-C.; Eu, J.-Y.; Lin, W.-L. Langmuir 1997, J3, 4018.

14. Noh, J.; Hara, M, Langmim 2000, 16, 2045.

15. Whelan, C. M.; Barnes, C. J.; Walker, C. G. H.; Brown, N. M. D. Surf. Sci. 1999, 425, 195.

16. Jung, H. H.; Won, Y. D.; Shin, S.; Kim, K. Langmitir 1999, 15, 1147.

17. Yang, G.; Quian, Y,; Engtrakul, C.; Sita, L. R.; Liu, G.-y. J. Phys. Chem. B 2000, j04, 9059 .

18. Noh, J.; Hara, M. Langmim 2001, 17, 7280.

19. Kakiuchi, T.; Iida, M.; Gon, N.; Hobara. D.; Imabayashi. S.-I.; Niki, K. Langntuir 2001, $17,1599$.

20. Noh, J. Bull. Korean Chem. Soc. 2005, 26, 553.

21. Kolega, R. R.; Schlenoff, J. B. Langmir 1998, 14, 5469.

22. Chen, S.; Li, L.; Boozer, C. L.; Jiang, S. L. Langmim 2001, 16, 9287. 